\title{
The cosmic-ray electron spectrum measured with the CALorimetric Electron Telescope
}

\author{
Lorenzo Pacini* \\ Institute of Applied Physics (IFAC-CNR), Via Madonna del Piano, 10, 50019, Italy \\ University of Florence, Via Sansone, 1 - 50019 Sesto, Fiorentino, Italy \\ National Institute for Nuclear Physics (INFN), Piazza dei Caprettari, 70 - 00186 Rome, Italy \\ E-mail: l.pacini@ifac.cnr.it
}

\section{for the CALET collaboration}

Direct measurements of cosmic-rays (CRs) are important to understand the sites and the processes of acceleration and propagation of high-energy particles in the interstellar medium. For example, detailed measurements of the high-energy electron+positron (hereafter simply "electron") spectrum can provide information about nearby CR sources. The electron spectrum may also exhibit features from Dark Matter (DM) annihilation. The CALorimetric Electron Telescope (CALET) is a Japanese-led international space mission promoted by JAXA (Japan Aerospace Exploration Agency) in collaboration with the Italian Space Agency (ASI) and NASA. The apparatus is continuously operating on board of the International Space Station (ISS) since October 2015. Its main objective is to perform precise direct measurements of the electron cosmic-ray spectrum in the energy region above $1 \mathrm{TeV}$; other scientific objectives are the measurement of hadron spectra, from proton to iron and above, up to several hundreds of $\mathrm{TeV}$ and the detection of gamma-ray emissions up to $10 \mathrm{TeV}$. The instrument consists of a deep homogeneous calorimeter, a sampling-imaging calorimeter and a charge detector. The electron measurement is characterized by excellent energy resolution (about $1 \%$ at $1 \mathrm{TeV}$ ) and good proton rejection power (about $10^{5}$ at $1 \mathrm{TeV}$ ). We will discuss the current status of the electron data analysis, mainly focusing on the electron/proton discrimination methods. Also, we will present a comparison between electron spectra measured by CALET and other experiments. The current CALET electron measurement will be extended to higher energies in the next years, with the increase of accumulated statistics.

The 39th International Conference on High Energy Physics (ICHEP2018)

4-11 July, 2018

Seoul, Korea

${ }^{*}$ Speaker. 


\section{The CALET experiment.}

The CALorimetric Electron Telescope (CALET) is a space borne calorimeter for the direct observation of cosmic-ray (CR) spectra. The apparatus is installed on board of the International Space Station and it is continuously taking data since October 2015[1]. The main scientific objective is the measurement of the CR electron+positron (hereafter simply "electron") spectrum up to $20 \mathrm{TeV}$. This observation provides unique information regarding the physics of $\mathrm{CR}$ sources nearby the Earth, e.g. Super Nova Remnants, since the range of electrons above $1 \mathrm{TeV}$ is about $1 \mathrm{kpc}$. Furthermore it is relevant for the search of dark matter signatures, especially

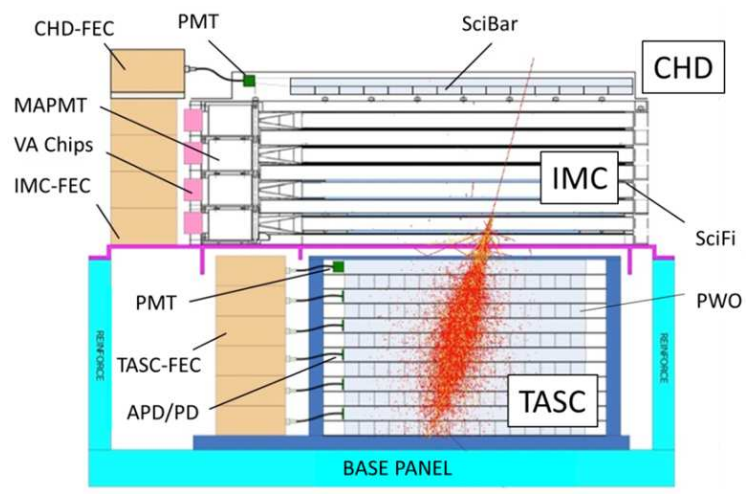

Figure 1: Drawing of the CALET main detector, an electromagnetic shower simulated with EPICS is also shown.

if it is combined with spectrometer measure-

ments of positron-electron ratio. Other CALET scientific goals are the measurement of proton and nuclei spectra up to several hundreds of $\mathrm{TeV}$ per nucleon and the identification of gamma rays with good angular resolution.

The CALET main detector is shown in fig. 1. It consists of 3 main sub-detectors. The CHarge Detector (CHD) is placed on top and made of 14 plastic scintillating paddles, each one read-out with a single PMT. Thanks to the high dynamic range, this instrument is capable of identifying the charge of incident nuclei, from proton to Iron and above. The IMaging Calorimeter (IMC) is composed by 8 layers of scintillating fibers separated by thin tungsten sheets. Thanks to the fine segmentation, this detector provides information about the direction of the incoming particle. The total IMC thickness is $3 \mathrm{X}_{0}$, thus it can be used to reconstruct the early stages of the shower development and identify the incident photon direction. The Total AbSorption Calorimeter (TASC), which consists of 12 layers of PWO bars, is the main sub-detector of CALET. Thanks to the large thickness, of about $27 \mathrm{X}_{0}$, the energy resolution for electrons is about $2 \%$ up to $20 \mathrm{TeV}$ [2]. A high dynamic range is achieved thanks to a PD-APD double read-out of the scintillation light and a double-gain front-end electronics. Beside the energy resolution, the TASC design achieves a good discrimination of electrons from the background, which mainly consists of protons, as discussed in what follows.

\section{Event selection for electrons.}

In this section a brief review of the analysis procedure used for the electron measurement is presented; more detailed descriptions are in [3] and [4].

The total CALET acceptance (or geometric factor) is about $1036 \mathrm{~cm}^{2} \mathrm{sr}$; a particle is in acceptance when it hits the 5th IMC layer, the TASC top layer, and has a shower length in TASC which is larger than $27 \mathrm{X}_{0}$. 

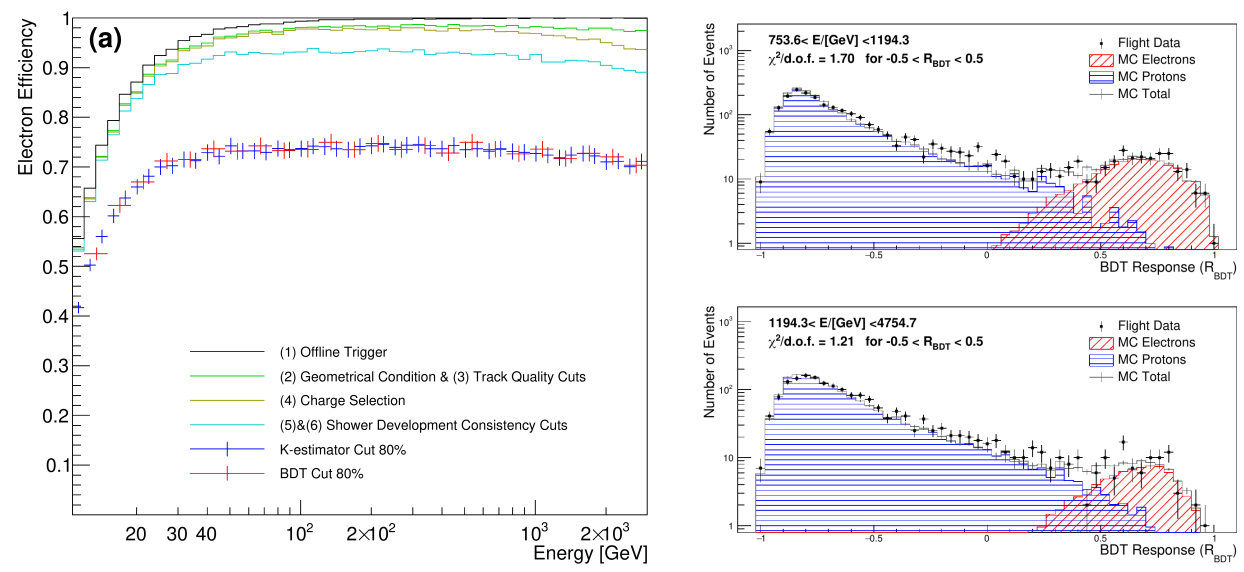

Figure 2: Left: event selection efficiencies obtained with the EPICS MC simulation as a function of the simulated electron energy. Right: BDT output distribution for electrons, protons and flight data in two energy bins, obtained with the EPICS MC simulation.

The first event selection is an Offline Trigger, which is similar to the hardware trigger but employing thresholds which are slightly higher than the real ones. It requires a large energy deposit in the last two IMC layers and first TASC layer; the efficiency for electrons is greater than $90 \%$ above $30 \mathrm{GeV}$ while it is about $30 \%$ for protons at $1 \mathrm{TeV}$.

A reconstructed track with the "ElectroMagnetic tracking algorithm"[5] in the nominal acceptance is then required. An additional event selection named "Track Quality Cut" rejects events when the reconstructed track is not consistent with the shower axis computed with the TASC energy deposits. To reject helium and heavier nuclei, a charge selection based on the combination of energy deposits in the CHD paddles and IMC fibers hit by the primary particle is employed.

Two "Shower Development Consistency Cuts" select events with energy deposit profile in TASC and IMC which is consistent with a typical electromagnetic shower. The first one is based on a log-likelihood cut regarding the energy deposit in TASC and IMC layers. The second one requires a large energy deposit in fibers nearby the reconstructed track in the last layer of the IMC.

At this stage of the analysis, the only considerable background is due to protons, since their abundance in $\mathrm{CR}$ above $1 \mathrm{TeV}$ is about $10^{3}$ times that of electrons. Two alternative proton rejection cuts have been developed; a detailed description of these selections is in [6]. The first one involves two variables: the fraction of energy deposited in the last layer of TASC, named $F_{E}$, and the lateral shower spread in the first layer of TASC, named $R_{E}$. To achieve the best rejection power, while keeping a high efficiency for electrons, the cut is applied on a single variable $(K)$ defined as $K=\log _{10}\left(F_{E}\right)+0.5 \cdot R_{E}$.

The second proton rejection cut involves a Multi-Variate Analysis (MVA) based on the Boosted Decision Trees (BDT) algorithm. The variables included in this analysis are the $F_{E}, R_{E}$, some parameters related to the longitudinal fit of the shower in TASC (by using a Gamma distribution) and IMC (by an exponential fit). The performance of the described event selection, e.g. efficiency and contamination due to protons, has been studied by using two independent MC simulations, based on EPICS and GEANT4 packages. The efficiency of each selection is shown in the left panel of 
fig. 2; above $30 \mathrm{GeV}$ both the rejection cuts ( $K$ and BDT) feature an efficiency of about $70 \%$. The right panels of fig. 2 show the response of the BDT algorithm obtained with EPICS in two high energy bins: the proton contamination remains below $20 \%$ in the entire energy range. These results are confirmed with the GEANT4 simulation and validated with precise comparisons between flight data and MC simulations, for example the right panels of fig. 2 show a good agreement between flight and MC data for the BDT response.

\section{Electron flux measured with CALET.}

The first observation the $\mathrm{CR}$ electron flux up to $3 \mathrm{TeV}$ with the CALET experiment was published in November 2017 [3], while an extended measurement up to $4.8 \mathrm{TeV}$ was published in June 2018 [3]. The full acceptance of the experiment and 780 days of data were used for this measurement, which is shown in fig. 3. There is a good agreement with the AMS-02 and PAMELA results below $1 \mathrm{TeV}$, but the DAMPE observation at about $1.5 \mathrm{TeV}$, which can be related to a peak in the spectrum, is not compatible with

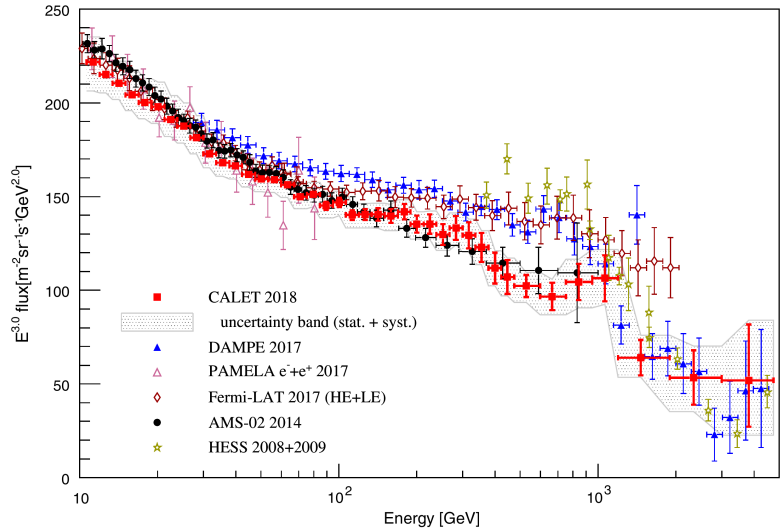

Figure 3: CR electron flux measured by CALET in comparison with other observations [4]. The vertical error bars represent the statistical errors, the uncertainty band indicates the quadratic sum of the statistical and systematic errors. CALET results at a level of $4 \sigma$.

Though the current statistics do not achieve a clear measurement of the spectral break observed by DAMPE, the CALET spectrum is consistent with this structure. The accuracy of the spectral break observation with CALET will improve in the next years by adding new flight data, i.e. reducing the statistical errors.

\section{References}

[1] Yoichi Asaoka et al. (CALET Collaboration), Astroparticle Physics 100 (2018) 29-37, DOI:10.1016/j.astropartphys.2018.02.010

[2] Yoichi Asaoka et al. (CALET Collaboration), Astroparticle Physics 91 (2017) 1-10, DOI:10.1016/j.astropartphys.2017.03.002

[3] O. Adriani et al. (CALET Collaboration), PRL 119, 181101 (2017), DOI:10.1103/PhysRevLett.119.181101

[4] O. Adriani et al. (CALET Collaboration), PRL 120, 261102 (2018), DOI:10.1103/PhysRevLett.120.261102

[5] Y. Akaike et al., Proc. Sci., ICRC2013 (2013) 726.

[6] L. Pacini, Y. Akaike et al., Proc. Sci., ICRC2017 (2017) 163. 